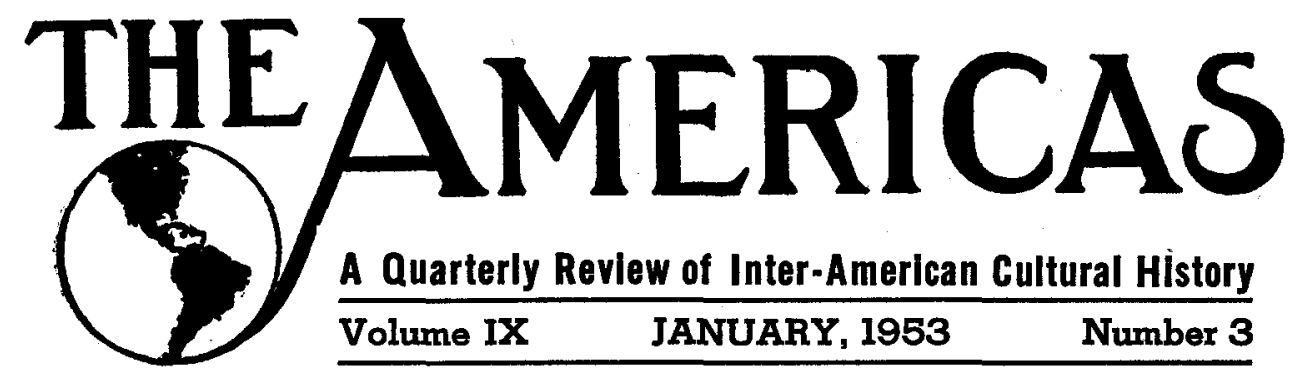

Teaching Latin-American Philosophy.....Arthur Berndtson 263

The Nacimiento in Mexico.......Manuel Romero de Terreros 273

A Sixteenth-Century German Colonizing Venture in VenezUELA............................... Lacas 275

Mary of Agreda and the Southwest United States

William H. Donabue

291

New Data Regarding the Origins of the Franciscan Missions in Peru, 1532-1569.............Lino G. Canedo

\title{
DOCUMENTS
}

A Group of Documents Concerning the Franciscan Missions in Peru, 1532-1569...... (Ed.) Lino G. Canedo 349

\section{INTER-AMERICAN NOTES}

A Columbus Letter.................Roscoe R. Hill 359

Rev. Dr. Ellis Makes Tour of South America........ 360

Library of Congress Appoints Dr. Howard F. Cline as

Director of Its Hispanic Foundatron.......... 361

Medina Centennial Observed on Two Continents.... 361

Serra Award of the Americas Bestowed on Dr. Victor Andrés Belaunde $\ldots \ldots \ldots \ldots \ldots \ldots \ldots \ldots \ldots, 362$

BOOK REVIEWS $\ldots \ldots \ldots \ldots \ldots \ldots \ldots \ldots \ldots \ldots \ldots, 363$ 


\section{EDITORIAL STAFF}

Francis B. Steck, O.F.M., Ph.D., Quincy College.

Maynard Geiger, O.F.M., Ph.D., Mission Archivist, Santa Barbara, California.

Lazaro Lamadrid, O.F.M., Lic.Hist., Washington, D. C.

Assistant Managing Editor

Bede A. Dauphinee, O.F.M., M.A,
Fidel Chauvet, O.F.M., Ph.D., Mexico City, D. F.

Lino G. Canedo, O.F.M., Ph.D., Archivo Ibero-Americano, Madrid, Spain.

Michael B. McCloskey, O.F.M., Siena College.

\section{Managing Editor}

Roderick WheEler, O.F.M., Ph.D., Academy of American Franciscan History. Academy of American Franciscan History.

象

\section{ADVISORY EDITORS}

Herbert E. Bolton, Ph.D., University of California.

Robert S. Chamberlain, Ph.D., Carnegie Institution of Washington.

Carlos E. Castaneda, Ph.D., University of Texas.

John Tate Lanning, Ph.D., Duke University.

J. Manuel Espinosa, Ph.D., Department of State, Washington, D. C.

France V. Scholes, Ph.D., University of New Mexico.

David Rubio, O.S.A., Ph.D., Madrid, Spain.

Enrique Carlos de la Casa, Ph.D.,

Secretary of the Inter-American Institute.

Manoez Cardozo, Ph.D.,

Oliveira Lima Library,

Catholic University of America.

Richard F. Pattee,

National Catholic Welfare Conference.
Lewis Hanke, Ph.D.,

Hispanic Foundation, Library of Congress.

IRENE A. WRIGHT, F.R.H.S., Department of State, Washington, D. C.

Leo Francis Stock, Ph.D.,

Carnegie Institution of Washington.

Ruth Lapham Butler, Ph.D.,

Newberry Library, Chicago.

Marie R. Madden, Ph.D.,

Teachers' Institute of Brooklyn.

Roscoe R. Hill, Ph.D.,

National Archives (retired).

Joseph F. ThorNing, Ph.D.,

Carrollton Manor, Md.

William F. Montavon, LL.D., Director, Legal Department, National

Catholic Welfare Conference.

Henry Grattan Doyle, Litt.D., Dean, George Washington University.

W. Eugene Shiels, S.J., Ph.D.,

Xavier University, Cincinnati, $O$.
Address all communications to

THE MANAGING EDITOR

29 Cedar Lane, N. W.,

Washington 14, D. C.
Entered as second class matter at the Postoffice at Washington, D. C., under the Act of March 3, 1879.

Additional entry at the Post Office at Richmond, Va.

Publisbed quarterly (July, October,

January and April). Annual subscription $\$ 4.00$ for four issues. 